\title{
Numerical and perturbation solutions for the gauss potential
}

\author{
Saad Naji Abood and Narjis Zamil Abdulzahra* \\ Department of Physics, College of Science, AL-Nahrain University, Baghdad, Iraq.
}

Global Journal of Engineering and Technology Advances, 2022, 10(02), 043-059

Publication history: Received on 01 January 2022; revised on 10 February 2022; accepted on 12 February 2022

Article DOI: https://doi.org/10.30574/gjeta.2022.10.2.0033

\begin{abstract}
We will focus on the Schrodinger eigenvalue problem for a Gauss potential in this study. When high and relatively large values of the coupling constant g2 are involved, we will compare eigenvalues E determined numerically with those obtained using the asymptotic series. However, we were interested in the mathematical elements of this comparison throughout the course of this work and explored it for considerably larger, albeit no longer physically plausible, values of g2. Even for power potentials where the Gaussian is a common example, Muller's perturbation method shows some fascinating mathematical characteristics of the Schrodinger equation. The solution's overall analytic features are very similar to well-known periodic differential equations like the Mathieu equation.
\end{abstract}

Keywords: Schrödinger Equation; Gauss Potential; Perturbation Theory; Schrodinger eigenvalue problem

\section{Introduction}

The theoretical laboratory of the Schrodinger equation and its solutions, both for the scattering as well as the bound states, have served an important purpose to shed some light on the much more complex phenomena of high energy particle collisions various potential functions were used in this equation where these potentials vary in their degree of singularities at the origin as well as at large distances. But ever since the field of potential scattering was recognized as a helpful testing ground for various particles physics model, one basic property of the solution was realized early. This was that very few potential models can have analytic form. This fact, however, is not uncommon in relativistic interactions.

The Yukawa potential $-g^{2} \exp (-A r) / r$ is most celebrated potential model for which the Schrodinger equation is not exactly solvable.

The Gauss potential $-g^{2} \exp \left(-A r^{2}\right)$ is another important potential, adapted first by Bethe and Bacher [1] to investigate the ground state of the deuteron. Here again we have another interesting physical situation where the Schrodinger equation cannot be solved exactly.

These two representatives of a large class of phenomenological physics potentials are heavily used in nuclear and particle physics. But the analytic properties of the solutions in the range or $r(0$ to $\infty)$ are taken for granted. For the singular potential models, though much more complicated than the regular models, the local and global existence of the solutions are discussed in the ref. [2]. In his papers, Muller [3, 4, 5] discussed some basic properties of the solutions by a power series perturbation method for these two potentials. Muller uses expansions in the domain of large coupling constants $g^{2}$. He also obtains asymptotic solutions of the S-wave radial Schrodinger equation for these potentials. The

\footnotetext{
${ }^{*}$ Corresponding author: Narjis Z Abdulzahra

Department of Physics, College of Science, AL-Nahrain University, Baghdad, Iraq. 
asymptotic are given in terms of parabolic cylinder functions and confluent hyper-geometric functions. Apart from the intrinsically interesting asymptotic solutions, Muller also obtained the associated expansions for the eigenvalues $E$.

As a rule, the perturbation techniques employ power series methods were the power series solution is given in terms of some convenient small parameter. Despite the fact that these series solutions usually are divergent, the approximate solution obtained by cutting off the formal series at some $\mathrm{i}^{\text {th }}$ term is quite adequate for a great many calculations. These solutions do not necessarily approach the exact solutions with increasing $i$; instead, for fixed $i$, it tends to the exact solution as the parameter approaches zero. We caution, however, that one should not accept the series solutions without verifying the range of validity first.

The scattering solutions have been amply discussed in literature. We intend to concentrate here on the Schrodinger eigenvalue problem for a Gauss potential. We shall compare eigenvalues $E$ calculated numerically with those obtained by the asymptotic series where large and moderately large values of the coupling constant $\mathrm{g}^{2}$ are involved. In the course of this work, however, we took an interest in the mathematical aspects of this comparison and accordingly studied it for much larger, though no longer physical realistic values of $\mathrm{g}^{2}$. We found it advantageous to use some modifications a very simple numerical procedure developed independently by Canosa and de Oliveira [6], Ixaru [7], and Gordon [8] rather than follow more conventional algorithms such as Numerov's step-by step scheme [9-12] or Rayleigh-Ritz [13] to solve the problem for the ground state $(L=0)$ and excited states. For the asymptotic expansion approach, we use the perturbation technique previously introduced by Muller [3]. Although Muller's eigenvalue expansion is the only expression of interest of interest here for computational purposes, we examine and present the details of the derivation of the eigenfunctions series expansion also.

It is noteworthy that Muller's perturbation method reveals some interesting mathematical features about the Schrodinger equation for even power potentials where the Gaussian is a typical example. The overall analytic characteristics of the solution are quite analogous to well-known periodic differential equations such as the Mathieu equation.

\section{Numerical Solution for the Schrodinger Equation for a Gauss Potential}

We consider the one-dimensional radial Schrodinger equation for a Gauss potential in the form:

$$
\frac{d^{2} \Psi}{d r^{2}}+(E-P(r)) \Psi(r)=0
$$

Where

$$
P(r)=\frac{L(L+1)}{r^{2}}-g^{2} \exp \left(-A^{2} r^{2}\right)=0
$$

Is referred to as the "potential energy" function. Clearly, this is just the centrifugal force term lumped with a Gauss potential. We solve this eigenvalue problem, (2.1), (2.2), with the boundary conditions $\Psi(0)=\Psi(\infty)=0$, numerically by the Canosa-de-Oliveira-Gordon-Ixaru (COGI) method.

The numerical algorithm has proved to be convenient and straightforward to carry out practice. In particular, a single computer program can be used to completely solve the problem, which is, any desired number of eigenvalues $E$, the corresponding normalized eigenfunctions $\Psi$, and their nodes for all allowed $L$ values.

\subsection{Formulation of the COGI Method}

Equation (2.1) with a potential function (2.2) which vanishes at infinity has, for large values of $r$, eigenfunctions $\Psi(r) \approx \exp (-r \sqrt{E})$ which reach infinitesimally small values. Therefore, we may use the approximate boundary conditions:

$$
\Psi(0)=0, \quad \Psi(R)=0
$$

For sufficiently large $R$. 
Central to implementing the COGI technique is the approximation of the potential P(r) by a step function with an arbitrary number of steps $n$ subintervals $0 \leq r \leq R$. There are an infinite variety of step functions which may be chosen to represent the potential over the $n$ subintervals $0 \leq r \leq r_{1}, r_{1} \leq r \leq r_{2}, \ldots, r_{n-1} \leq r \leq r_{n}=R$. Canosa and de Oliveira [6] and Canosa [7] used constant steps for their sample problems while Gordon [8, 14] used a linear reference potential. The former results in simple trigonometric and hyperbolic solutions while the latter involves Airy functions or Bessel functions of order $1 / 3$, since COGI has been shown to be a second order method for a constant as well as a linear step function [7], we follow Canosa and de Oliveira formulation and represent (2.2) by a step function with constants $p_{1}, p_{2}, \ldots, p_{n}$ appropriately defined in each corresponding subinterval. We need not have each subinterval of equal width. This permits us the flexibility of approximating the potential the potential for a given number of steps $n$ with more steps near its minimum where it varies rapidly and thereby yields a better representation of the potential. However, note that no matter what step function is selected we can define the approximate problem to be as close as we wish to the exact problem by taking a sufficiently large number of steps.

In each step, the resulting differential equation for any eigenvalue $E$

$$
\frac{d^{2} \Psi}{d r^{2}}+\left(E-P_{i}\right) \Psi(r)=0 \quad i=0,1,2, \ldots, n
$$

Has constant coefficients and is integrated exactly in terms of hyperbolic or trigonometric functions. Thus, in each step $i \neq 1$ or $n$, the solution to Eq. (2.4) is

$$
\Psi=A_{i} C\left(\beta_{i} r\right)+B_{i} S\left(\beta_{i} r\right) \quad \alpha_{i} \equiv E-P_{i}, \beta_{i} \equiv \sqrt{\left|\alpha_{i}\right|} . i=2,3, \ldots, n-1 \ldots
$$

Where $\mathrm{A}_{\mathrm{i}}, \mathrm{B}_{\mathrm{i}}$ are integration constants and $\mathrm{C}$ and $\mathrm{S}$ are circular cosine and sine for $\alpha_{i}>0$ and hyperbolic cosine and sine for $\alpha_{i}<0$. So that the boundary conditions (2.3) are satisfied, the solutions for $i=1$ and $i=n$ are respectively.

$$
\Psi=B_{1} S\left(\beta_{1} r\right), \quad \Psi=B_{n} S\left(\beta_{n}(r)+B_{i} S\left(\beta_{i}(r-R)\right)\right.
$$

With $\mathrm{B}_{1}, \mathrm{~B}_{\mathrm{n}}$ integration constants, therefore, once the eigenvalues $E$ and the integration constants are determined, the problem is solved.

We obtain the eigenvalues and corresponding eigenfunctions by matching the solution (2.5) and (2.6) and its derivative at the interval boundaries. In this way, we derive a homogenous system of $2 n-n$ equations for the $2 n-2$ integration constants.

We write this system in the following form in matrix notation.

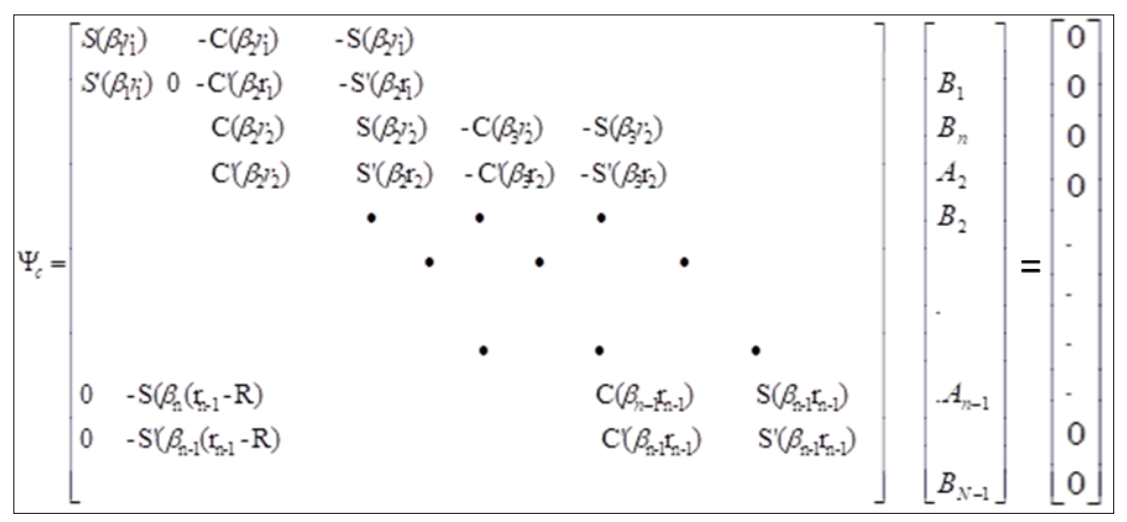


It is well known that the determinant of $\Psi$ must vanish for the existence of a nontrivial solution. There are an infinite number of constants $\beta_{i}$ which are related to the eigenvalues of our approximate problem as defined by (2.5) and (2.6). This is guaranteed because (2.4) and (2.3) form a Strum-Liouville system, which is known to have an infinite number of eigenvalues.

Instead of solving $\operatorname{det} \Psi=0$ with $\Psi$ in the form of (2.7) for the eigenvalues, it is numerically advantageous to first perform some elementary matrix operations on $\Psi$ and group the elements of $\Psi$ in $2 \times 2$ matrices, getting

$$
\begin{aligned}
\operatorname{det}(\Psi) & =\operatorname{det}\left(\Psi_{n-1, n-1}\right) \times \ldots \times \operatorname{det}\left(\Psi_{2,2}\right) \\
& \times \operatorname{det}\left(\Psi_{1,1}+\Psi_{1,2} \Psi_{2,2}^{-1} \Psi_{2,3}^{-1} \Psi_{3,3}^{-1} \ldots \Psi_{n-2, n-1} \Psi_{n-1, n-1}^{-1} \Psi_{n-1,1}\right) \\
& =0
\end{aligned}
$$

Where

$$
\begin{aligned}
& \Psi_{1,1}=\left[\begin{array}{ll}
S\left(\beta_{1} r_{1}\right) & 0 \\
S^{\prime}\left(\beta_{1} r_{1}\right) & 0
\end{array}\right] \ldots \ldots . . .(2.9 \mathrm{a}) \\
& \Psi_{n-1,1}=\left[\begin{array}{ll}
0 & -S\left(\beta_{n}\left(r_{n-1}-R\right)\right) \\
0 & -S^{\prime}\left(\beta_{n}\left(r_{n-1}-R\right)\right)
\end{array}\right] \\
& \Psi_{i, j}=\left[\begin{array}{ll}
C\left(\beta_{j} r_{j}\right) & -S\left(\beta_{j} r_{i}\right) \\
C^{\prime}\left(\beta_{j} r_{j}\right) & -S^{\prime}\left(\beta_{j} r_{i}\right)
\end{array}\right]
\end{aligned}
$$

It is apparent from (2.9c) that $\operatorname{det}\left(\Psi_{i, i}\right)=1, i=1,2,3, . ., n-1$. Now, by making one more simplification where the elementary addition formulas of the circular and hyperbolic sine and cosine are used to multiply the $2 \times 2$ matrices of the form $\Psi_{i-1,} \Psi_{i, i}^{-1}$ in Eq.(2.8) together so that, explicitly,

$$
{ }^{-} \Psi_{i-1, i}=\Psi_{i-1, i} \Psi_{i, i}^{-1}=\left\{\begin{array}{lr}
{\left[\begin{array}{lr}
\cosh \left(\beta_{i}\left(r_{i}-r_{i-1}\right)\right) & -\beta_{i}^{-1} \sinh \left(\beta_{i}\left(r_{i}-r_{i-1}\right)\right) \\
-\beta_{i} \sinh \left(\beta_{i}\left(r_{i}-r_{i-1}\right)\right) & \cosh \left(\beta_{i}\left(r_{i}-r_{i-1}\right)\right)
\end{array}\right]} \\
{\left[\begin{array}{lr}
\cos \left(\beta_{i}\left(r_{i}-r_{i-1}\right)\right) & -\beta_{i}^{-1} \sin \left(\beta_{i}\left(r_{i}-r_{i-1}\right)\right) \\
\beta_{i} \sin \left(\beta_{i}\left(r_{i}-r_{i-1}\right)\right) & \cos \left(\beta_{i}\left(r_{i}-r_{i-1}\right)\right)
\end{array}\right]}
\end{array}\right\} \ldots
$$

We will have the actual expression to be used to obtain the eigenvalues. This eigenvalue equation is

$$
\operatorname{det}(\Psi)=\operatorname{det}\left(\Psi_{1,1}+\Psi_{1,2}^{-} \Psi_{2,3}^{-} \ldots \Psi_{n-2, n-1}^{-} \Psi_{n-1,1}\right)=0
$$

Which represents a reduction in the evaluation of the determination of the original $(2 n-2) \times(2 n-2)$ matrix to that of $2 \times 2$ matrix in Eq.(2.11). It is useful to consider the determinant in Eq. (2.11) simply as a function $F$ whose arguments $E_{\text {appro. }}$. will be approximations to the energy eigenvalues for the bound states, 


$$
F\left(E_{\text {approx. }}\right)=\operatorname{det}(\Psi)
$$

We derive an even simpler formula for obtaining the corresponding eigenfunctions. Consider again system (2.7), but now with the elements grouped in $2 \times 2$ matrices defined by (2.9) along with their associated two-row vectors

$$
c_{1}=\left[\begin{array}{l}
B_{1} \\
B_{2}
\end{array}\right], \quad c_{i}=\left[\begin{array}{l}
A_{i} \\
B_{i}
\end{array}\right], \quad i=2,3, \ldots, n-1
$$

In this way

$$
\begin{array}{cc}
\Psi_{1,1} c_{1}-\Psi_{1,2} c_{2} & =0 \\
\Psi_{2,2} c_{2}-\Psi_{2,3} c_{3} & =0 \\
\cdot & \\
\cdot & \Psi_{n-2, n-2} c_{n-2}-\Psi_{n-2, n-1} c_{n-1}=0 \\
& +\Psi_{n-1, n-1} c_{n-1}=0
\end{array}
$$

For each eigenvalue determined from (2.11) the matrices $\Psi_{i, j}$ are known explicitly in (2.14). Hence, we need only determine the elements in the vectors $c_{1}$. Our problem (2.1) - (2.3) has only one arbitrary constant $B_{1}$ or $B_{n}$ available to start the integration so we must either solve from the last equation to the first in (2.14) or vice versa. By choosing some value for $\mathrm{B}_{\mathrm{n}}$, we have

$$
\begin{aligned}
& c_{n-1}=-\Psi_{n-1, n-1}^{-1} \Psi_{n-1,1} c_{1} \\
& c_{n-2}=-\Psi_{n-2, n-2}^{-1} \Psi_{n-2, n-1} \Psi_{n-1, n-1}^{-1} \Psi_{n-1,1} c_{1} \\
& \cdot \cdot \cdot \cdot \cdot \cdot \cdot \cdot \cdot \Psi_{n-1, n-1}^{-1} \Psi_{n-1,1} c_{1}
\end{aligned}
$$

The last equation in (2.15), for $m=2$, determines $c_{2}$, and from this and the first equation in (2.14) we could, in principle, obtain the integration constant $\mathrm{B}_{1}$ to entirely solve the problem. However, stability difficulties were encountered resulting in unsatisfactory eigenfunctions values near the tail end of the interval $0 \leq r \leq R$ when we used this procedure in solving completely in one direction.

We correct this situation by the process of "matching in the middle". Sine $\mathrm{B}_{1}$ is available to start our integration in the first equation in (2.14) and $B_{2}$ to start at the last, we solve from both ends until a convenient matching point is reached in the middle. Possible matching points have been proposed by, for example, Blatt [9], Canosa and de Olivera [6], Coley [10], Fox [12], and Froese [11]. Each suggestion was made dependent on either the behaviour of $\mathrm{P}(\mathrm{r})$ or 
$\alpha(r)=E=P(r)$. The actual choice s not critical for a single equation since we simply want to reduce the unstable accumulation of error during the integration in either direction. Following Fox [12] and Froese [11], we choice the location of the last zero of $\alpha(r)$ as our matching point. This effectively reduces the source of our difficulties for it is here that exponential type behaviour of $\Psi(r)$ begins and proceeds for the remainder of the interval. The eigenfunctions computed in the forward integration will differ from those of the backward by a factor. They are adjusted to match by multiplying each set by its reciprocal value at the middle.

With $\mathrm{m}=$ matching point, we rewrite $(2.15)$ as

$$
\begin{gathered}
c_{n-1}=-\Psi_{n-1, n-1}^{-1} v_{n-1}, v_{n-1} \equiv \Psi_{n-1,1} c_{1}, \\
c_{n-2}=-\Psi_{n-2, n-2}^{-1} v_{n-2,} v_{n-2} \equiv \Psi_{n-2, n-1} \Psi_{n-1, n-1}^{-1} v_{n-1}, \\
\cdot \cdot \cdot \cdot \\
c_{m}=-\Psi_{m, m}^{-1} v_{m}, \ldots v_{m} \equiv \Psi_{m, m+1} \Psi_{m+1, m+1}^{-1} v_{m+1} .
\end{gathered}
$$

Except for the first matrix in the right hand side, $\Psi_{n-1,1}$, it is numerically convenient to group he others in Eq.(2.17) in pairs as

$$
\Psi_{i-1, i}^{b}=\Psi_{i-1, i} \Psi_{i, i}^{-1}=\left[\begin{array}{lr}
\cosh \left(\beta_{1}\left(r_{i}-r_{i-1}\right)\right) & -\beta_{i}^{-1} \sinh \left(\beta_{i}\left(r_{i}-r_{i-1}\right)\right) \\
-\beta_{i} \sinh \left(\beta_{i}\left(r_{i}-r_{i-1}\right)\right) & \cosh \left(\beta_{i}\left(r_{i}-r_{i-1}\right)\right)
\end{array}\right]
$$

Only hyperbolic functions are involved since $\alpha_{i}<0$ for all $i=n-1, n-2, . ., m$. From Eqns. (2.5), (2.13) and (2.16) we can write the eigenfunction expressions in vector notation as

$$
\Psi(r)=-\Psi_{i, i}^{-1} v_{i} . f(r), \quad f(r)=\left[\begin{array}{c}
C\left(\beta_{i} r\right) \\
S\left(\beta_{i} r\right)
\end{array}\right] i \neq n
$$

Equivalently, by using the elementary addition formulas for the hyperbolic and trigonometric functions in evaluating the inner product in Eq.(2.18), we have the desired eigenfunction formula

$$
\Psi^{b}(r)=\left(-1 / \beta_{i}\right)\left\{\beta_{i} v_{i 1} \cosh \left(\beta_{i}\left(r_{i}-r\right)\right)-v_{i 2} \sinh \left(\beta_{i}\left(r_{i}-r\right)\right)\right\}
$$

Where $v_{i 1}$ and $v_{i 2}$ are the first and second elements, respectively, in the column vector v1 defined in Eq.(2.16). We emphasize, however, that Eq.(2.19) is valid only for backwards integration and $i \neq n$. .

The corresponding eigenfunction formulas for forward integration of Eq.(2.14) are

$$
\begin{aligned}
& \Psi^{f}(r)=\left\{\left(1 / \beta_{i}\right)\left\{\beta_{i} v_{i 1} \cosh \left(\beta_{i}\left(r_{i-1}-r\right)\right)-v_{i 2} \sinh \left(\beta_{i}\left(r_{i-1}-r\right)\right)\right\}, \alpha_{i}<0\right. \\
& \Psi^{f}(r)=\left\{\left(1 / \beta_{i}\right)\left\{\beta_{i} v_{i 1} \cos \left(\beta_{i}\left(r_{i-1}-r\right)\right)-v_{i 2} \sin \left(\beta_{i}\left(r_{i-1}-r\right)\right)\right\}, \alpha_{i}>0\right.
\end{aligned}
$$

Where $i \neq 1$ and $\mathrm{v}_{\mathrm{i} 1}$ and $\mathrm{v}_{\mathrm{i} 2}$ are now determined from 


$$
\begin{aligned}
& v_{2} \equiv \Psi_{1,1} c_{1}, \\
& v_{2} \equiv \Psi_{2,2} \Psi_{1,2}^{-1} v_{2}, \\
& \cdot \cdot \cdot \\
& v_{m} \equiv \Psi_{m-1, m-1} \Psi_{m-2, m-2}^{-1} v_{m-1},
\end{aligned}
$$

With numerical considerations again dictating that we first group all the matrices, except for the initial one, in Eq.(2.21) in pairs as

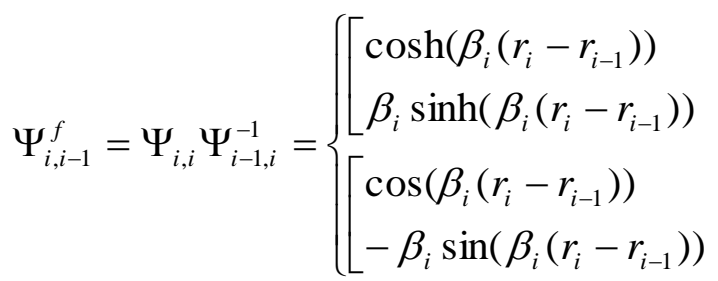

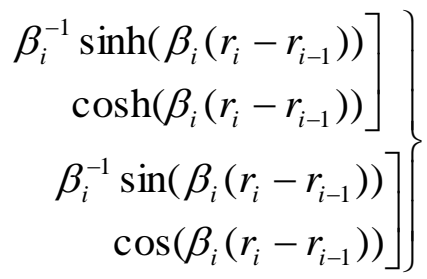

A complete derivation of Eq. (2.20) has been given previously by Canosa and de Oliveira [6].

\subsection{Computational Details}

We summarize the computational procedures used to solve the Schrodinger equation for a Gauss potential. These are:

- Chose an interval length $0 \leq r \leq R$ reasonably approximating the boundary conditions. We found that $R=5$ was adequate as the eigenfunctions already began approaching zero asymptotically.

- Approximate $P(r)$ in Eq. (2.1) with a suitably convenient step function $P(r)$ for an appropriate number of steps $n(=1000$ and/or 500 in all cases considered $)$ over the interval.

We approximate $\frac{L(L+1)}{r^{2}}-g^{2} \exp \left(A^{2} r^{2}\right)$ by the following step functions:

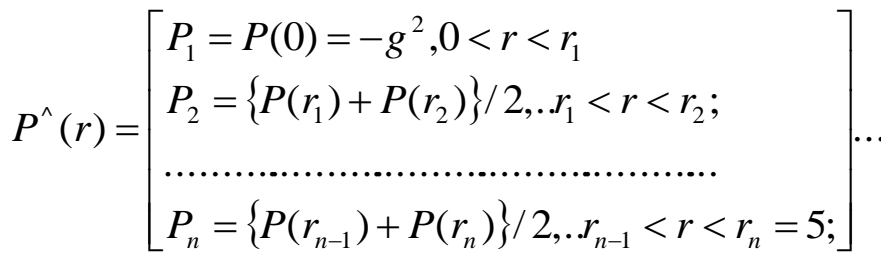

For $\mathrm{L}=0$ where each step is of equal width $h=r_{i}-r_{i-1}$;

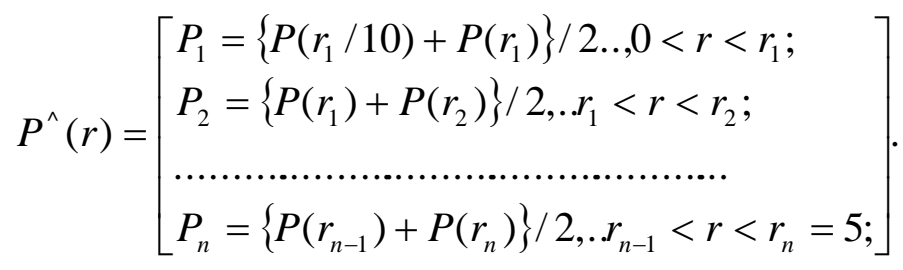

For $L>0$ where each step is of equal width $h=r_{i}-r_{i-1}$; 


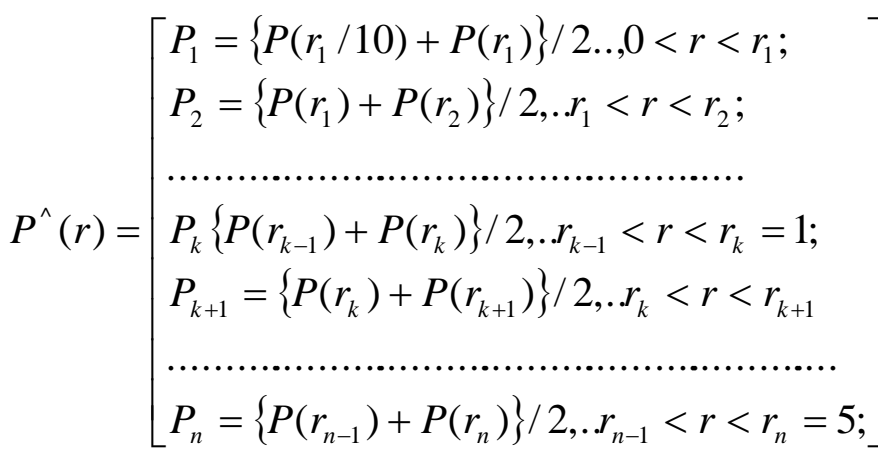

For $L>0$ where $k=n / 2$ step sizes are of width $h_{1}=1 / n$ on $0<r<1$ and $\mathrm{k}$ step sizes are of width $h_{1}=4 / n$ on $0<r<5$. Two separate programs were written; one, using step functions (2.23) and (2.24); the other, (2.23) and (2.25) to solve for all allowed L values. In each instance, these calculations are stored to be used in the remaining steps.

\subsubsection{Determination of the eigenvalues from the eigen values formula}

(2.11). No initial guesses are required for the eigenvalues in contrast to the conventional numerical methods mentioned earlier. We need only specify approximately where they lie, subdivide this range into a predetermined number of points, and substitute each of these endpoints of the sub-range as argument in (2.11), or (2.12). We obtained all desired eigenvalues by using the range

$$
\begin{aligned}
& 0 \geq E_{\text {approx. }} \geq \frac{4}{5} P_{\min }(r), \quad L=0, \\
& 0 \geq E_{\text {approx. }} \geq \frac{3}{4} P_{\min }(r), \quad L>0,
\end{aligned}
$$

Whenever a change of sign of $F\left(E_{\text {approx. }}\right)$ at two successive points is detected, we obtain the root (eigenvalue) by Muller's iteration method [15]. The eigenvalues obtained are stored.

- Choose a convenient matching point. Our choice of the last zero of $\alpha(r)$ makes our program quite general as it will handle any value of $\mathrm{L}$ and $\mathrm{E}$. We deduce this by noting that the behavior of $\alpha(r)$ for $\mathrm{L}=0$ and $L>0$ are quite similar. When the angular momentum term is absent we simply bypass that section of our algorithm concerned with $\Psi(r)$ initially negative.

- Determination of the eigenfunctions by Eq. (2.19) or Eq. (2.20) depending on the direction of integration, for each eigenvalue found in step $\mathrm{C}$. We choice $\mathrm{B} 1=\mathrm{B} 2=1$ to start our forward and backward integration. The vector VI given by Eq. (2.16) or Eq. (2.21) are calculated and stored first. For each set of the eigenfunctions calculated, we matched them in the middle by the procedure given in the discussion earlier.

- Determination of the nodes of the eigenfunctions corresponding to the jth eigenvalues. Muller's iteration method is again used whenever a change of sign of the eigenfunction at two successive values of $r$ is detected. Since the jth eigenvalues has $\mathrm{j}-1$ nodes [12] we use this calculation as a check to make no desired eigenvalues are omitted.

It is worth mentioning that the present method allows us a computer program which may be applied equally well to the Schrödinger equation potentials other than the Gaussian potential considered in this study with only minor changes.

\section{Muller's Solution by Asymptotic Expansions}

Throughout this section we consider a Gauss_potential expanded as a power series in r²: 


$$
V(r)=-g^{2} \sum_{j=2}^{\infty} \frac{\left(-A^{2}\right)^{j}}{j !} r^{2 j}
$$

\subsection{Asymptotic Solution near the origin}

Muller' [3] derived asymptotic expansion solutions of the Schrodinger equation for a Gauss potential, (2.1) and (2.2), in terms of (A) parabolic cylinder functions for $\mathrm{L}=0$, and (B) confluent hypergeometric, functions for $L \geq 0$. Both eigenfunctions obtained are valid for $r$ near the origin. A similar derivation of the asymptotic expansion of the Jost solution was also necessary in order to obtain an eigenfunctions that would be valid for large $r$. Then by analytic continuation of the solutions in their common range of validity a solution valid over the entire interval is obtained.

Here, we reproduce Muller's result (B) and in the process we will end up with a useful eigenvalues can be calculated. We note that the perturbation method used to derive that Jost solution also leads us to the same expression from which we can calculate the eigenvalues. However, it is less desirable to arrive at eigenvalue expression in this way because it is more tedious.

With Eq.(3.1) we write the radial Schrodinger equation for a Gauss potential as

$$
\frac{d^{2} \Psi}{d r^{2}}+\left(E-\frac{L(L+1)}{r^{2}}+g^{2}-g^{2} A^{2} r^{2}\right) \Psi=-g^{2} \sum_{j=2}^{\infty} \frac{\left(-A^{2}\right)^{j}}{j !} r^{2 j} \Psi
$$

Changing the independent variable to $z=(2 g A)^{1 / 2} r$ as

$$
\frac{d^{2} \Psi}{d z^{2}}+\left(\frac{E+g^{2}}{2 g A}-\frac{L(L+1)}{z^{2}}-\frac{1}{4} z^{2}\right) \Psi(r)=-\frac{1}{2} \sum_{j=2}^{\infty}\left(\frac{A}{g}\right)^{j-1} \frac{\left(-\frac{1}{2} z^{2}\right)^{j}}{j !} \Psi
$$

Now for large values of the coupling constants $\mathrm{g}^{2}$, the right hand side of Eq.(3.3) is $O(1 / g)$. Hence for $|g| \rightarrow \infty$, the radial wave equation may be approximated by

$$
\frac{d^{2} \Psi_{0}}{d z^{2}}+\left(\frac{E+g^{2}}{2 g A}-\frac{L(L+1)}{z^{2}}-\frac{1}{4} z^{2}\right) \Psi_{0}=0
$$

This Eq.(3.4) may be transformed into a standard mathematical form by the substitutions

$$
\begin{aligned}
& \Psi_{0}(z)=z^{L+1} \exp \left(-\frac{1}{4} z^{2}\right) x_{0}(z), \\
& S=\frac{1}{2} z^{2} \ldots \ldots \ldots \ldots(3.5 \mathrm{~b})
\end{aligned}
$$

Yielding as the differential equation for $x_{0}(z)$

$$
S \frac{d^{2} x_{0}}{d S^{2}}+(b-S) \frac{d x_{0}}{d S}-a x_{0}=0
$$

Where

Where 


$$
\begin{aligned}
& a=\frac{1}{2}\left(1+\frac{3}{2}\right)+\left(E+g^{2}\right) / 4 g A, \\
& b=L+\frac{3}{2} \ldots \ldots \ldots \ldots . . .(3.7 \mathrm{~b})
\end{aligned}
$$

Eq.(3.6) is readily identified as a confluent hypergeometric equation whose solution is [16]

$$
x_{0}(z)=M(a, b ; S)
$$

Where M is the confluent hypergeometric function, hence, the solution of Eq.(3.4) is

$$
\Psi_{0}(z)=z^{L+1} \exp \left(-\frac{1}{4} z^{2}\right) M\left(a, b, \frac{1}{2} z\right)
$$

Or for convenience later, we let

$$
\Psi(a, b ; z)=\Psi_{0}(z)
$$

$\Psi_{0}$ will be normalizable bound state wavefunction if

$$
a=-n \text { for } n=0,1,2, \ldots
$$

Then from Eq. (3.7a) and setting $q=4 n+3$ we have for Eq. (3.4)

$$
E=-g^{2}+g A(2 L+q)
$$

Or for

$$
E=-g^{2}+g A(2 L+q)-2 A^{2} \Delta
$$

Where $\Delta$ is an expansion of $O(1 / g)$.

To obtain Muller's solution to Eq.(3.3) we subtract Eq.(3.13) into Eq.(3.3), thus deriving the fundamental equation

$$
D_{q} \Psi(z)=\left[-\frac{1}{2} \Delta h+\frac{1}{4} \sum_{i=2}^{\infty} \frac{h^{i-1}}{i !}\left(\frac{1}{2} z^{2}\right)^{i}\right] \Psi(z)
$$

With

$$
D_{q} \Psi(z)=\frac{1}{2}\left[\frac{d^{2}}{d z^{2}}+L+\frac{1}{2} q-\frac{L(L+1)}{z^{2}}-\frac{1}{4} z^{2}\right] \ldots \ldots \ldots \ldots
$$

$h=-A / g$

To which the perturbation method is applied. Following the procedure shown by Eqns. (3.16)-(3.26) in ref.[3] successive contributions $\Psi^{(i)}, i=1,2, \ldots$, to the solution $\Psi(z)$ of Eq.(3.14) are made. Each of $\Psi^{(i)}$ leaves uncompensated a sum of terms on right hand side of Eq.(3.14) from which the next contribution $\Psi^{(i+1)}$ is dominated. This expression obtained by adding all $\Psi^{(i)}$, s together is 


$$
\Psi(z)=\Psi(a, b ; z)+\sum_{i=1}^{\infty} h^{i} \sum_{j=2 i}^{\infty} P_{i}(a, j) \Psi(a+j, b ; z)
$$

With coefficients $P_{i}(a, \pm j)$ defined by, for example,

$$
\begin{aligned}
& P_{i}(a, 2)=\frac{1}{16} a(1+a) \\
& P_{1}(a,-2)=-\frac{1}{16}\left(a^{2}-a+2 a b+b+b^{2}\right) \\
& P_{1}(a, 1)=\frac{1}{8}\left(2 a b-2 a-4 a^{2}\right) \\
& P_{1}(a,-1)=-\frac{1}{8}\left(6 a b-4 a^{2}+2 a-2 b-4 b^{2}\right)
\end{aligned}
$$

Expansion Eq.(3.17) is the eigenfunction for Eq.(3.14) if the sum of all remaining uncompensated terms is set equal to zero. Explicitly, we have

$$
\begin{aligned}
& 0= h\left[-\frac{1}{2} \Delta+\frac{1}{2^{6}}\left(3\left(q^{2}+1\right)+4(3 q-1) L+8 L^{2}\right)\right] \\
& h^{2}\left[\begin{array}{l}
\frac{1}{2^{3} 3}\left(30 a^{2} b-20 a^{3}-12 a b^{2}-6 a b-4 a+3 b^{2}+2 b+b^{3}\right. \\
+\frac{1}{2^{6}}\left(68 a^{3}-102 a^{2} b+10 a+18 a b+42 a b^{2}-5 b-9 b^{2}-4 b^{3}\right.
\end{array}\right] \\
&+\ldots .
\end{aligned}
$$

Solving for $\Delta$ in Eq.(3.19), substituting into Eq.(3.13) and simplifying we thus obtain the desired eigenvalue expansion

$$
\begin{aligned}
E= & -g^{2}+g A(2 L+q)-\frac{A^{2}}{2^{4}}\left[3\left(q^{2}+1\right)+4(3 q-1) L+8 L^{2}\right] \\
& -\frac{A^{3}}{3.2^{8}}\left[q\left(11 q^{2}+1\right)+2\left(33 q^{2}-6 q+1\right) L+24(5 q-1) L^{2}+64 L^{3}\right] \\
& -\frac{A^{3}}{3.2^{15}}\left[\begin{array}{l}
4\left(85 q^{4}+2 q^{2}-423\right)+L\left(2720 q^{3}-71 q^{2}+32 q+2976\right) \\
+32 L^{2}\left(252 q^{2}-12 q+64\right)+256 L^{3}(41 q-9)+4096 L^{4}
\end{array}\right]+O\left(1 / g^{3}\right)
\end{aligned}
$$

For large values of the coupling constant g $^{2}$, Eq.(3.20) is a proper asymptotic expansion with rapidly decreasing terms.

\subsection{Connection of results with expansion in terms of parabolic cylinder functions}

We note that, as expected, eigenvalues expansion (3.20) with $\mathrm{L}=0$ becomes 


$$
\begin{aligned}
& E=-g^{2}+g g-\frac{3 A^{2}\left(q^{2}+1\right)}{2^{4}}+\frac{q\left(11 q^{2}+1\right) A^{2}}{2^{6} .3} h \\
&-\frac{\left(85 q^{4}+2 q^{2}-423\right)}{2^{9} \cdot 3} h^{2}+O\left(h^{3}\right)
\end{aligned}
$$

By setting $h=-A / 4 g$, this is Eq.(2.25) in [3] even though Muller derived this letter expression independently by considering the zero angular momentum solution of the Schrodinger equation in terms of parabolic cylinder functions.

We remark finally that Muller's eigenfunction of the Schrodinger equation for a Gauss potential with zero angular momentum is

$$
\Psi(z)=\Psi_{q}(z)+\sum_{i=1}^{\infty} h^{i} \sum_{j=-2 i}^{2 i} P_{i}(q, 4 j) \Psi_{q+4 j}(z)
$$

With $\Psi_{q}$ now the parabolic cylinder function and $P_{i}(q, 4 j)$ defined as

$$
\begin{aligned}
& P_{1}(q, \pm 4 j)=\mp \frac{1}{32}\left(4 q^{2} \pm 20 q+24\right) \\
& P_{1}(q, \pm 8)=\mp \frac{1}{64}\left(q^{2} \pm 10 q+21\right)
\end{aligned}
$$

\section{Computed Results and Discussion}

We considered terms up to $O\left(1 / g^{2}\right)$ whenever Muller's eigenvalue expansion Eqns.(3.20) or (3.21) was used. All calculations tabulated below assume the mass of the exchange particle $\mathrm{A}^{2}$ to be one.

\subsection{Eigenvalues Calculated for $L=0$}

We present in Table (1) the eigenvalues $E_{0}^{C O G I}$ and $E_{0}^{M}$ computed, respectively, by the numerical method of section 2 and the perturbation method of section 3 which correspond to the energy levels associated with a bound system where centrifugal force does not act on the particles. Such a system can have only one energy level, the ground state $\mathrm{E}_{0}$. Both procedures produced this value (with q $=3$ in Eq.(3.20) or eq. (3.21)) for each coupling constant considered.

A comparison of the eigenvalues computed by the two methods for each $\mathrm{g}^{2}$ was made. The absolute difference $\delta=E_{0}^{C O G I}-E_{0}^{M}$ for each pair of values was calculated and is listed in the last column of Table 1. Clearly, this shows that agreement between the eigenvalues of the COGI and Muller's procedure improve $\mathrm{g}^{2}$ increases.

\subsection{Eigenvalues Calculated for $L=1$}

Table 2 shows the energy eigenvalues $u^{E^{C O G I}}, v^{E^{C O G I}}$ and $\mathrm{E}^{\mathrm{M}}$ obtained using, respectively, uniform step function (2.24), variable step function (2.25), and Muller's (3.20). Although they physical system now being considered permits energy levels higher than the ground state, we find that with a Gauss potential there are no excited states. In fact, we obtained eigenvalues corresponding to the ground state only for large values of the coupling constant.

The differences $\delta_{u} E=u^{E^{C O G I}}-E^{M}$ and $\delta_{v} E=v^{E^{C O G I}}-E^{M}$ are again calculated to measure the closeness between the eigenvalues obtained by the two methods. They don't agree very well. However, the observation we made in the preceding subsection concerning the relationship between $\mathrm{g}^{2}$ and $\delta$ appear to remain valid. In other words $\mathrm{g}^{2}$ must be 
extremely large for this case before Muller's expansion approaches the numerically computed eigenvalues (see Tables 3 and 4).

\subsection{Eigenvalues corresponding to very large $\mathrm{g}^{2}$}

Table 3 and Table 4 gives the eigenvalues obtained for $\mathrm{L}=0$ and $\mathrm{L}=1$, respectively, when very large though physically insignificant $\mathrm{g}^{2}$ are considered. It is noteworthy that the higher eigenvalues calculated with Muller's expansion tend to those obtained with the COGI procedure in a manner exactly like that for the lowest $\mathrm{E}_{0}$.

\subsection{Eigenfunctions calculated by COGI}

Numerical values of the eigenfunction $\Psi$ at selected points calculated by the COGI method are reported in Tables 5, 6, 7 , 8, and 9. Each set of values correspond to a coupling constant $g^{2}=16$ and either $\mathrm{L}=0$ or $\mathrm{L}=1$. Tables 5 and 6 show the zero angular momentum case with step size $h=0.01$ and $h=0.005$ used in the calculations, respectively. Tables 7,8 and 9 considers the case $\mathrm{L}=1$ with these same uniform step sizes used for determining $\Psi$ in Table 7 and 9 respectively. Values in Table 8 were computed using variable step sizes $h_{1}=0.004$ on $0 \leq r \leq 1$ and $h_{2}=0.016$ on $1 \leq r \leq 5$. We illustrate graphically the eigenvalues in Tables and using the values listed in Tables 4 and 5 . The Tables behaviour shown is typical for the eigenfunctions obtained for all other coupling constant as well.

\section{Concluding Remarks}

The calculations indicate that at least for $\mathrm{L}=0$ cases both methods mentioned in this study give very good results in approximating the solution of the Schrodinger equation for a Gauss potential.

We have reason to believe that the COGI solution is more accurate than Muller's for all cases considered, however. The numerical method has been applied previously too many problems similar in behaviour to the present one, but with known exact solutions [6,7] and the results obtained were always very accurate. COGI is therefore, a reliable method, at least for problems of the present type.

Table 1 Comparison of Eigenvalues computed by COGI and Muller's method for L = 0

\begin{tabular}{|c|c|c|c|c|}
\hline g2 & steps in COGI & $E_{0}^{C O G I}$ & $E_{0}^{M(a)}$ & $\delta=E_{0}^{C O G I}-E_{0}^{M}$ \\
\hline 6.0 & 500 & -0.7379340 & -0.7299476 & -0.0079864 \\
\hline 7.0 & 500 & -1.130848 & -1.123055 & -0.007793 \\
\hline 8.0 & 500 & -1.567733 & -1.560781 & -0.006952 \\
\hline 9.0 & 500 & -2.040577 & -2.034505 & -0.006072 \\
\hline 10.0 & 500 & -2.543395 & -2.538058 & -0.005337 \\
\hline 11.0 & 500 & -3.071584 & -3.066872 & -0.004712 \\
\hline 12.0 & 500 & -3.621620 & -3.6174428 & -0.004192 \\
\hline 13.0 & 500 & -4.190721 & -4.186965 & -0.003756 \\
\hline 14.0 & 500 & -4.776641 & -4.773258 & -0.003383 \\
\hline 15.0 & 500 & -5.377546 & -5.374485 & -0.003061 \\
\hline 15.0 & 1000 & -5.377587 & -5.364485 & -0.003120 \\
\hline 16.0 & 500 & -5.991916 & -5.989136 & -0.002780 \\
\hline 16.0 & 1000 & -5.991967 & -5.989136 & -0.002831 \\
\hline
\end{tabular}

(a) $q=3$ for all eigenvalues $E^{\mathrm{M}}$ calculated with Eq. (3.21). 
Table 2 Comparison of Eigenvalues $u^{E^{C O G I}}, v^{E^{C O G I}}$ with EM for L $=1$

\begin{tabular}{|c|c|c|c|c|c|c|}
\hline g2 & $\begin{array}{l}\text { Steps in } \\
\text { COGI }\end{array}$ & $u^{E^{C O G I}}$ & $v^{E^{C O G I}}$ & EM (a) & $\delta_{u} E=u^{E^{C O G I}}$ & $\delta_{v} E=v^{E^{C O G I}}$ \\
\hline 14.0 & 500 & -0.4263363 & \multirow[t]{2}{*}{-0.4269721} & -0.2567219 & -0.1696144 & \multirow[t]{2}{*}{-0.1702502} \\
\hline 14.0 & 1000 & -0.4269381 & & -0.2567219 & -0.1702162 & \\
\hline 15.0 & 500 & -0.7197134 & \multirow[t]{2}{*}{-0.7205267} & -0.5751195 & -0.1445939 & \multirow[t]{2}{*}{-0.1454072} \\
\hline 15.0 & 1000 & -0.7204489 & & -0.2567219 & -0.1453294 & \\
\hline 16.0 & 500 & -1.042534 & \multirow[t]{2}{*}{-1.043505} & -0.9177456 & -0.124789 & \multirow[t]{2}{*}{-0.125760} \\
\hline 16.0 & 1000 & -1.043405 & & -0.9177456 & -0.125660 & \\
\hline
\end{tabular}

(a) $\mathrm{q}=3$ in Eq. (3.21) for all eigenvalues $\mathrm{E}^{\mathrm{M}}$ calculated.

Table 3 Difference between Eigenvalues $u^{E^{C O G I}}$ and EM for very large g2, $\mathrm{L}=0^{\text {(a) }}$

\begin{tabular}{|l|l|l|l|l|}
\hline g2 & $\delta E_{0,3}^{(b)}$ & $\delta E_{1,7}$ & $\delta E_{2,11}$ & $\delta E_{3,15}$ \\
\hline 25.0 & -0.00130 & -0.3204714 & - & - \\
\hline 40.0 & -0.00026 & -0.125567 & - & - \\
\hline 50.0 & -0.00017 & -0.8317 & (c ) & - \\
\hline 75.0 & 0.00101 & -0.04045 & -0.554289 & - \\
\hline 100.0 & 0.00156 & -0.02437 & -0.1260 & (d) \\
\hline
\end{tabular}

(a) All eigenvalues calculated COGI involved $\mathrm{n}=500$ steps.

(b) $E_{i}^{C O G I}-E_{i}^{M} \quad i=i^{\text {th }}$ Eigenvalues; j=values of q used in Eq.(3.21)

(c) $E^{C O G I}=0.1973158 ; E^{M}=+1.196081$ (Unexpected)

(d) $E^{C O G I}=1.197602 ; E^{M}=+1.038106$ (Unexpected)

Table 4 Difference between Eigenvalues $u^{E^{C O G I}}$ and $E^{M}$ for very large $\mathrm{g}^{2}, \mathrm{~L}=1^{\text {(a) }}$

\begin{tabular}{|c|c|c|c|}
\hline \multicolumn{1}{|l|}{$\mathbf{g}^{2}$} & $\delta E_{0,3}^{(b)}$ & $\delta E_{1,7}$ & $\delta E_{2,11}$ \\
\hline 25.0 & -0.050047 & - & - \\
\hline 50.0 & -0.00629 & -0.393194 & - \\
\hline 75.0 & 0.00681 & -0.16188 & - \\
\hline 100.0 & 0.01527 & -0.8091 & -0.872626 \\
\hline
\end{tabular}

(a) Step function (2.24) with $\mathrm{n}=500$ steps was used in calculating all eigenvalues by COGI method.

(b) $\delta E_{i, j}=E_{i}^{C O G I}-E_{i}^{M}, \mathrm{i}=\mathrm{i}^{\text {th }}$ Eigenvalues; $\mathrm{j}=$ value of q used in Eq. (3.20) 
Table 5 Eigenfunction $\Psi$ calculated by COGI with $n=500$ steps, L = 0, g2 = 16

\begin{tabular}{|c|c|c|c|}
\hline$r$ & $\Psi(r)$ & $r$ & $\Psi(r)$ \\
\hline 0.0 & 0.0 & 2.6 & $0.27152432 \mathrm{D}-01$ \\
\hline 0.1 & $0.42196916 \mathrm{D} 00$ & 2.7 & $0.21259947 \mathrm{D}-01$ \\
\hline 0.2 & $0.80304015 \mathrm{D} 00$ & 2.8 & $0.16645126 \mathrm{D}-01$ \\
\hline 0.3 & $0.11099086 \mathrm{D} 01$ & 2.9 & $0.13031484 \mathrm{D}-01$ \\
\hline 0.4 & $0.13222685 \mathrm{D} 01$ & 3.0 & $0.10202062 \mathrm{D}-01$ \\
\hline 0.5 & $0.14347150 \mathrm{D} 01$ & 3.1 & $0.79867705 \mathrm{D}-02$ \\
\hline 0.6 & $0.14551345 \mathrm{D} 01$ & 3.2 & $0.62523362 \mathrm{D}-02$ \\
\hline 0.7 & $0.14006684 \mathrm{D} 01$ & 3.3 & $0.48943702 \mathrm{D}-02$ \\
\hline 0.8 & $0.12928195 \mathrm{D} 01$ & 3.4 & $0.38311213 \mathrm{D}-02$ \\
\hline 0.9 & $0.11530952 \mathrm{D} 01$ & 3.5 & $0.29985717 \mathrm{D}-02$ \\
\hline 1.0 & $0.10000000 \mathrm{D} 01$ & 3.6 & $0.23465903 \mathrm{D}-02$ \\
\hline 1.1 & 0.84933259D 00 & 3.7 & $0.18359172 \mathrm{D}-02$ \\
\hline 1.2 & $0.70788080 \mathrm{D} 00$ & 3.8 & $0.14358006 \mathrm{D}-02$ \\
\hline 1.3 & $0.5809065 \mathrm{D} \quad 00$ & 3.9 & $0.11221462 \mathrm{D}-02$ \\
\hline 1.4 & $0.47105028 \mathrm{D} 00$ & 4.0 & $0.87606634 \mathrm{D}-03$ \\
\hline 1.5 & $0.37839294 \mathrm{D} 00$ & 4.1 & $0.68274220 \mathrm{D}-03$ \\
\hline 1.6 & $0.30177893 \mathrm{D} 00$ & 4.2 & $0.53053208 \mathrm{D}-03$ \\
\hline 1.7 & $0.23937566 \mathrm{D} 00$ & 4.3 & $0.41027004 \mathrm{D}-03$ \\
\hline 1.8 & $0.18911793 \mathrm{D} 00$ & 4.4 & $0.31471403 \mathrm{D}-03$ \\
\hline 1.9 & $0.18497968 \mathrm{D} 00$ & 4.5 & $0.23810977 \mathrm{D}-03$ \\
\hline 2.0 & $0.11711932 \mathrm{D} 00$ & 4.6 & $0.17584423 \mathrm{D}-03$ \\
\hline 2.1 & 0.91941029D -01 & 4.7 & $0.12416784 \mathrm{D}-03$ \\
\hline 2.2 & 0.72105350D-01 & 4.8 & $0.79968715 \mathrm{D}-03$ \\
\hline 2.3 & $0.56512384 \mathrm{D}-01$ & 4.9 & $0.40585218 \mathrm{D}-03$ \\
\hline 2.4 & $0.44272646 \mathrm{D}-01$ & 5.0 & $0.4058218 \mathrm{D}-03$ \\
\hline 2.5 & $0.346744230 \mathrm{D}-01$ & & \\
\hline
\end{tabular}

Table 6 Eigenfunction $\Psi$ calculated by COGI with $n=1000$ steps, $L=0, g^{2}=16$

\begin{tabular}{|l|l|l|l|}
\hline$r$ & $\Psi(r)$ & $r$ & $\Psi(r)$ \\
\hline 0.0 & 0.0 & 3.0 & $0.10077474 \mathrm{D}-01$ \\
\hline 0.5 & $0.14237401 \mathrm{D} 01$ & 3.5 & $0.29618933 \mathrm{D}-02$ \\
\hline 1.0 & $0.99238016 \mathrm{D} 00$ & 4.0 & $0.86520530 \mathrm{D}-03$ \\
\hline 1.5 & $0.37418591 \mathrm{D} 00$ & 4.5 & $0.23467554 \mathrm{D}-03$ \\
\hline 2.0 & $0.11570976 \mathrm{D} 00$ & 5.0 & 0.0 \\
\hline 2.5 & $0.34251716 \mathrm{D}-01$ & & \\
\hline
\end{tabular}


Table 7 Eigenfunction $\Psi$ calculated by COGI with $\mathrm{n}=500$ steps, $\mathrm{L}=1, \mathrm{~g} 2=16$

\begin{tabular}{|l|l|l|l|}
\hline$r$ & $\Psi(r)$ & $r$ & $\Psi(r)$ \\
\hline 0.0 & 0.0 & 2.6 & $0.27830849 \mathrm{D} 00$ \\
\hline 0.1 & $0.46501485 \mathrm{D}-01$ & 2.7 & $0.2485308 \mathrm{D} 00$ \\
\hline 0.2 & $0.179270050 \mathrm{D} 00$ & 2.8 & $0.22184999 \mathrm{D} 00$ \\
\hline 0.3 & $0.37547531 \mathrm{D} 00$ & 2.9 & $0.19811871 \mathrm{D} 00$ \\
\hline 0.4 & $0.60410791 \mathrm{D} 00$ & 3.0 & $0.17692314 \mathrm{D} 00$ \\
\hline 0.5 & $0.83267262 \mathrm{D} 00$ & 3.1 & $0.15796708 \mathrm{D} 00$ \\
\hline 0.6 & $0.10335823 \mathrm{D} 00$ & 3.2 & $0.14098953 \mathrm{D} 00$ \\
\hline 0.7 & $0.11881991 \mathrm{D} 01$ & 3.3 & $0.12576032 \mathrm{D} 00$ \\
\hline 0.8 & $0.12879994 \mathrm{D} 01$ & 3.4 & $0.11207611 \mathrm{D} 00$ \\
\hline 0.9 & $0.13333453 \mathrm{D} 01$ & 3.5 & $0.99756875 \mathrm{D}-00$ \\
\hline 1.0 & $0.13308877 \mathrm{D} 01$ & 3.6 & $0.88642824 \mathrm{D}-01$ \\
\hline 1.1 & $0.12906505 \mathrm{D} 01$ & 3.7 & $0.78591691 \mathrm{D}-01$ \\
\hline 1.2 & $0.12235436 \mathrm{D} 01$ & 3.8 & $0.96476423 \mathrm{D}-01$ \\
\hline 1.3 & $0.11396490 \mathrm{D} 01$ & 3.9 & $0.61183149 \mathrm{D}-01$ \\
\hline 1.4 & $0.10473002 \mathrm{D} 01$ & 4.0 & $0.53609415 \mathrm{D}-01$ \\
\hline 1.5 & $0.95317184 \mathrm{D} 00$ & 4.1 & $0.46662631 \mathrm{D}-01$ \\
\hline 1.6 & $0.86136058 \mathrm{D} 00$ & 4.2 & $0.40258717 \mathrm{D}-01$ \\
\hline 1.7 & $0.77423844 \mathrm{D} 00$ & 4.3 & $0.34320895 \mathrm{D}-01$ \\
\hline 1.8 & $0.69331240 \mathrm{D} 00$ & 4.4 & $0.28778615 \mathrm{D}-01$ \\
\hline 1.9 & $0.61959006 \mathrm{D} 00$ & 4.5 & $0.23566593 \mathrm{D}-01$ \\
\hline 2.0 & $0.55288042 \mathrm{D} 00$ & 4.6 & $0.18623939 \mathrm{D}-01$ \\
\hline 2.1 & $0.49302411 \mathrm{D} 00$ & 4.7 & $0.13893365 \mathrm{D} 0-1$ \\
\hline 2.2 & $0.43955767 \mathrm{D} 00$ & 4.8 & $0.93204478 \mathrm{D}-01$ \\
\hline 2.3 & $0.39192387 \mathrm{D} 00$ & 4.9 & $0.4852953 \mathrm{D}-01$ \\
\hline 2.4 & $0.34954124 \mathrm{D} 00$ & 5.0 & 0.0 \\
\hline 2.5 & $0.31184496 \mathrm{D} 00$ & & \\
\hline & & & \\
\hline & & \\
\hline & & \\
\hline & & 001 \\
\hline
\end{tabular}

Table 8 Eigenfunction $\Psi$ calculated by COGI with $n=500$ (variable) step size

\begin{tabular}{|l|l|l|l|}
\hline$r$ & $\Psi(r)$ & $r$ & $\Psi(r)$ \\
\hline 0.0 & 0.0 & 0.7 & $0.12059387 \mathrm{D} 01$ \\
\hline 0.1 & $0.58866508 \mathrm{D}-01$ & 0.8 & $0.2991245 \mathrm{D} 01$ \\
\hline 0.2 & $0.20095835 \mathrm{D} 00$ & 0.9 & $0.13382690 \mathrm{D} 01$ \\
\hline 0.3 & $0.40321926 \mathrm{D} 00$ & 1.0 & $0.13306013 \mathrm{D} 01$ \\
\hline 0.4 & $0.63398006 \mathrm{D} 00$ & 1.8 & $0.70471121 \mathrm{D} 01$ \\
\hline 0.5 & $0.86095765 \mathrm{D} 00$ & 2.6 & $0.28283470 \mathrm{D} 01$ \\
\hline 0.6 & $0.10574299 \mathrm{D} 00$ & 3.4 & $0.11382782 \mathrm{D} 00$ \\
\hline
\end{tabular}


Table 9 Eigenfunction $\Psi$ calculated by COGI with $n=1000$ step, L=1, g2=16

\begin{tabular}{|l|l|l|l|}
\hline$r$ & $\Psi(r)$ & $r$ & $\Psi(r)$ \\
\hline 0.0 & 0.0 & 3.0 & $0.17581877 \mathrm{D} 00$ \\
\hline 0.5 & $0.81842056 \mathrm{D} 00$ & 3.5 & $0.99679816 \mathrm{D}-01$ \\
\hline 1.0 & $0.13249084 \mathrm{D} 01$ & 4.0 & $0.53198912 \mathrm{D}-01$ \\
\hline 1.5 & $0.95315132 \mathrm{D} 00$ & 4.5 & $0.23542563 \mathrm{D}-01$ \\
\hline 2.0 & $0.54960487 \mathrm{D} 00$ & 5.0 & 0.0 \\
\hline 2.5 & $0.31171617 \mathrm{D} 00$ & & \\
\hline
\end{tabular}

\section{Compliance with ethical standards}

\section{Acknowledgments}

We thank to Prof. Saad A. Hamada for useful discussions and the communication of results prior to publication.

\section{Disclosure of conflict of interest}

No conflict of interest.

\section{References}

[1] HA Bethe, RF Bacher, 'Nuclear Physics Part A" Stationary States of Nuclei, Review of Modern Physics. 1936; 8: 82-229.

[2] HH Aly, F Odeh. Lett. El Nuovo Cim. 1971; 2: 193.

[3] HJW Muller. "Perturbation Theory for large Coupling Constants Applied to the Gauss Potential" Journal of Mathematical Physics. 1979; 11: 355-364.

[4] HJW Muller, K Schilcher. Jour. of Math. Phys. 1968; 9: 255.

[5] Perturbation Approach for Regular Interactions, Lectures in Theoretical High Energy Physics, H. H. Aly ed., Interscience, New York. 1968; 371-421.

[6] J Canosa, RJ de Oliveira. "A New method for the Solution of the Schrodinger Equation" Journal of Computational Physics. 1970; 5: 188-207.

[7] J Canosa. "Numerical Solution of Mathieu's equation" Journal of Computational Physics. 1971; 7: $255-272$.

[8] RG Gordon. "New Method for Constructing Wavefunctions for Bound States and Scattering" Journal of Chemical Physics. 1969; 5: 14-25.

[9] J Blatt. "Particle Point Concerning the Solution of the Schrodinger Equation" Journal of Computational Physics. 1967; 1: 382-396.

[10] JW Cooley. "An Improved Eigenvalues Corrector Formula for Solving the Schrodinger Equation for Central Field" Mathematics of Computations. 2005; 15: 363-374.

[11] C Froese. "On Solving $y^{\prime \prime}=f y+g$ with a Boundary Condition at Infinity" Mathematic of Computations. 2019; 16: 492-494.

[12] L Fox. "Numerical Solution of Ordinary and Partial Differential Equations" Pergamon Press, London. 1999.

[13] G Strang, G Fix. “An Analysis of the Finite Element Method" Prentice Hall. 1974.

[14] R Gordon." Quantum Scattering Using Piecewise Analytic Solutions" Methods in Computational Physics" B. Adler, S. Fernbach and M. Rotenberg, eds., Academic Press, New York. 2018; 11: 81-109.

[15] DE Muller. "A Method for Solving Algebraic Equations Using an Automatic Computer" Mathematics Tables and Other Aids to Computations. 2010; 10: 208-215.

[16] M Abramowitz, IA Stegum. eds., Handbook of Mathematical Functions Applied Mathematics Series no. 55, U. S. Dept. of Commerce, National Burden of Standards, Washington, and D. C. 1964. 